\title{
KIC 10001893: A pulsating sdB star with multiple trapped modes
}

\author{
M. Uzundag, ${ }^{1 \star}$ A.S. Baran, ${ }^{2}$ R.H. Østensen, ${ }^{3}$ M.D. Reed, ${ }^{3}$ J.H. Telting, ${ }^{4}$ \\ and B.K. Quick ${ }^{3}$ \\ ${ }^{1}$ Ankara Üniversitesi, Fen Bilimleri Enstitüsü, Astronomi ve Uzay Bilimleri Anabilim Dalı, İran Baştuğ Caddesi 06110 Dışkapı, Ankara, Turkey \\ ${ }^{2}$ Uniwersytet Pedagogiczny, Obserwatorium na Suhorze, ul. Podchorażych 2, 30-084 Kraków, Polska \\ ${ }^{3}$ Department of Physics, Astronomy, and Materials Science, Missouri State University, Springfield, MO 65897, USA \\ ${ }^{4}$ Nordic Optical Telescope, Rambla José Ana Fernández Pérez 7, 38711 Breña Baja, Spain
}

Accepted XXX. Received YYY; in original form ZZZ

\begin{abstract}
KIC 10001893 is a V1093 Her type pulsating subdwarf-B star, which was observed extensively by the Kepler spacecraft. It was a part of the survey searching for compact pulsators in the Kepler field of view. An amplitude spectrum of the star demonstrates a rich content of g-modes between 102 and $496 \mu \mathrm{Hz}$ as well as a few p-modes above $2000 \mu \mathrm{Hz}$. In total, we found 110 frequencies. The g-mode region contains 104 frequencies, while the p-mode region contains just six, altogether indicating the hybrid nature of KIC 10001893. The main goal of our analysis was to identify the detected modes and to find some features, which will significantly help modeling of the star. We found no multiplets, which points at a pole-on orientation, however, we defined modal degrees and relative radial orders using asymptotic period spacing. Eventually, we assigned 32 dipole $l=1$ and 18 quadrupole $l=2$ modes. The most remarkable feature we found are trapped modes, which are clearly seen in a reduce period diagram. It is the first time that three trapped modes are detected in one pulsating sdB star. Since the more trapped modes we find, the better sounding of the stellar interior we can do, this feature provides important constraints on the physical structure of the star. Mode trapping is likely caused by the He-H transition region and therefore it provides crucial constraints for making realistic theoretical models of hot subdwarfs.
\end{abstract}

Key words: subdwarfs, stars: oscillations (including pulsations)

\section{INTRODUCTION}

Subdwarf B (sdB) stars are located between the horizontal branch (HB) and the white dwarf cooling track, on the so-called extreme horizontal branch (EHB, Heber 2016). The sdB stars are compact objects with masses typically around 0.5 solar masses and radii between 0.15 and 0.35 solar radii. Observed effective temperatures range from about 20,000 to $40,000 \mathrm{~K}$. These stars are core He-burning with thin hydrogen envelopes $\left(M_{e n v}<0.01 M_{\odot}\right)$. Such a small mass of the hydrogen envelope does not allow sdB stars to ascend the asymptotic giant branch. Therefore, after all helium is exhausted in their cores, $\mathrm{sdB}$ stars move directly to the white dwarf cooling track. The most enigmatic part of this evolution is the envelope-stripping phase, during which almost the entire hydrogen envelope is removed. This must occur close to the tip of the RGB, just before the occurrence of the helium flash, after which the envelope contracts while the core expands, and before the star settles down in its stable helium-burning phase. The cause of the envelope stripping is most likely mass transfer involving a close

^ E-mail: Murat.Uzundag@ankara.edu.tr companion to an sdB star (Han et al. 2002), or mass loss from a significantly enhanced stellar wind (Yong et al. 2000).

The first pulsating sdB was discovered by a South African group of astronomers (Kilkenny et al. 1997) and enables us to probe the interiors of $\mathrm{sdB}$ stars using asteroseismology. It is a powerful tool, which allows us to sound stellar structure using natural vibrations. Pulsating sdB (sdBV) stars are classified as V361 Hya or V1093 Her stars based on their periods. The former class is dominated by p-mode frequencies higher than $2000 \mu \mathrm{Hz}$ while the latter class is dominated by g-modes, which are usually below $1000 \mu \mathrm{Hz}$. Some sdBV stars show both kinds of modes and are therefore called hybrid stars (Baran et al. 2005; Schuh et al. 2006). The first theoretical models of sdBV stars was a paper by Charpinet et al. (1996) followed by (e.g. Charpinet et al. 1997, 2000; Fontaine et al. 2003). A successful application of those models can be found in a number of papers (e.g. Koen et al. 1999; Charpinet et al. 2011; Van Grootel et al. 2013).

During the last six years significant headway has been made in the field of sdBV stars. The Kepler spacecraft delivered unprecedented data revealing features that were rarely or never seen in ground-based data. These features include rotational multiplets (e.g. Baran 2012), asymptotic period spacing (e.g. Reed et al. 
2011), Doppler beaming (e.g. Telting et al. 2012), and mode trapping (Østensen et al. 2014; Foster et al. 2015; Kern et al. 2017).

The Kepler observations made it possible to resolve the gmode region in the V1093 Her stars, something that, so far, has not been possible with ground-based data. This had profound impact on the theoretical models, where physics related to mixing (especially diffusion and overshooting) are poorly constrained. The first results by Reed et al. (2011) indicated that the asymptotic sequences were much smoother than predicted by the early theoretical works, in which stratification played a strong role and produced a sharp boundary in the $\mathrm{H} / \mathrm{He}$ transition zone. Such sharp boundaries are very efficient in producing trapped modes, and it was speculated that the absence of trapped modes might indicate that stronger diffusion could reduce the impact of this boundary. However, Charpinet et al. (2014) demonstrated that while the boundary indeed produces significant trapping at low radial order, modes of higher order (which are the ones that appear with high amplitude in the observations) become less sensitive to this transition layer as their wavelengths become long compared to the size of the boundary. But this discussion was abruptly turned on its head with the first discovery of trapped modes in KIC 10553698 A by $\varnothing$ stensen et al. (2014). With such a clear signature of mode trapping it became possible for the modelers to test the adequacy of their models in reproducing the observed features. The first result was reported already by Constantino et al. (2015), where they explored models with different degrees of core overshooting parameters and found that their models could reproduce similar trapping effects caused by the sharp composition gradient at the edge of the partially mixed zone associated with the $\mathrm{C}-\mathrm{O} / \mathrm{He}$ boundary outside the convective inner core. More recently, Ghasemi et al. (2017) explored models with various combinations of overshooting and diffusion parameters with the particular aim of matching the trapping patterns seein in KIC 10553698A. In their scenarios with small and moderate overshooting the mixing leads to the emergence of convective shells around the core which produces mode trapping patterns that have comparable trapping signatures to those observed in KIC 10553698A.

In this paper we present our analysis of KIC 10001893, which was observed with the Kepler spacecraft. It was confirmed as a V1093 Her pulsator with one month of data (Q3.3) during the Kepler survey phase (Østensen et al. 2010, 2011; Baran et al. 2011). Baran et al. (2011) found 27 frequencies, mostly in the g-mode region, with asymptotic sequences as reported by Reed et al. (2011). Silvotti et al. (2014) used data covering Q6-Q17.2 (1051 days), which is the entire (continuous) data coverage available. However, they concentrated only on the lowest frequency region, below the so-called cut-off frequency and interpreted three frequencies, found in that region, as indications of exoplanets.

Here we have used the same 3-year dataset as Silvotti et al. (2014), and we provide a detailed analysis of the frequencies above the cut-off frequency. The increased span of the observations gives better resolution and lowers the detection limit compared to Baran et al. (2011), allowing us to detect more frequencies. We apply seismic tools to the pulsations to identify features useful for constraining models, in particular the échelle and reduced period diagrams. Our mode identifications seem to be reasonable, even though multiplets are absent, and the detection of trapped modes is particularly noteworthy.

\section{PHOTOMETRIC DATA}

KIC 10001893 was observed by the Kepler spacecraft. The observations started on 24 June 2010 and finished on 11 May 2013, which covered Q6 to Q17.2. We downloaded all available data from the "Barbara A. Mikulski Archive for Space Telescopes" (MAST) ${ }^{1}$. The Kepler spacecraft has two types of exposure times, which are short-cadence (SC, $\sim 1 \mathrm{~min}$ ), and long-cadence (LC, $\sim 30 \mathrm{~min}$ ). We used short-cadence (SC) data which covers the frequency range up to the Nyquist at $8495 \mu \mathrm{Hz}$, and assuring both $p$ - and $g$-mode regions are covered. The MAST database provides data processed in a couple of different ways (RAW, SAP, PDCSAP). We used fluxes with the pre-search data conditioning (PDCSAP) module and widely known as PDC fluxes. These fluxes are corrected for systematic errors and contamination from nearby stars, while the other fluxes are not. We clipped data at $4 \sigma$ and de-trended each monthly chunk of data separately. Finally, we stitched the monthly chunks together making the data ready for Fourier analysis.

\section{FOURIER ANALYSIS}

We used a Fourier technique to calculate the amplitude spectrum from Q6-17.2 data and we show it in Fig. 1. The frequency resolution equals $0.0162 \mu \mathrm{Hzas}$ defined by $1.5 / \mathrm{T}$, where $\mathrm{T}$ is the time coverage of the data. We first tried the pre-whitening technique to fit and remove frequencies, however, profiles of signals at many frequencies were complex and we were unable to fit those. Therefore, we decided to determine the frequencies by-eye. We applied a 5$\sigma$ detection threshold (Baran et al. 2015a) of 0.02 ppt. We checked the detected frequencies against the list of artifacts (Baran 2013). Eventually, we found 110 significant peaks above the detection threshold, showing KIC 10001893 to be a rich g-mode pulsator with most pulsations below $500 \mu \mathrm{Hz}$ but significant frequencies all the way up to $3897 \mu \mathrm{Hz}$. We list all detected frequencies in Table 1.

\section{1 p-modes}

We found six frequencies in the high-frequency region. This area is relatively sparse in frequencies, with all of them located between 2880 and $3900 \mu \mathrm{Hz}$. We show a close-up of this region in Fig. 2. The highest amplitude is $0.07 \mathrm{ppt}$. We assigned those frequencies with p-modes. No multiplets are present in this region, and since such features are one of the most useful tools to make mode identifications, we were left with no chance to directly identify individual modes. The multiplets can also be useful in estimating stellar rotation. An absence of split modes can be caused by low $\mathrm{S} / \mathrm{N}$ in this region or if the stellar rotation axis is pointed towards the observer, which efficiently suppresses the amplitudes of the side components, or if the rotation rate is so slow that the span of the data is insufficient to resolve the multiplets. More details will be given in Section 4. A few frequencies beyond $4000 \mu \mathrm{Hz}$ are of instrumental origin, related to the widely known LC-readout time.

\section{2 g-modes}

We show the g-mode region of the amplitude spectrum in Fig. 3. This region is rich in frequencies with relatively large amplitudes compared to the p-modes (see Fig. 1). The frequencies with the

\footnotetext{
1 archive.stsci.edu
} 


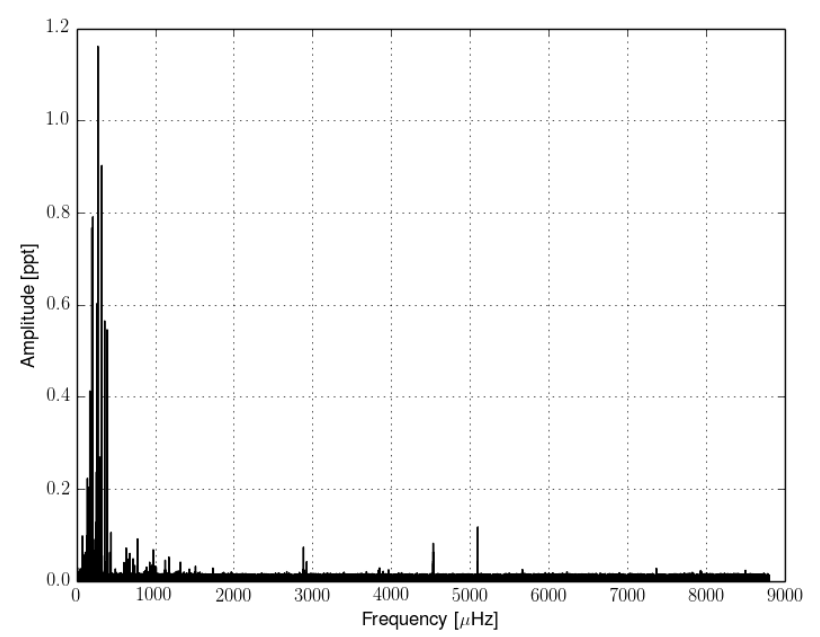

Figure 1. Amplitude spectrum, calculated up to the Nyquist frequency, showing both g- and p-modes. Close-ups of both regions are shown in Figs. 2 and 3.

highest amplitudes are located below $400 \mu \mathrm{Hz}$. The region above $500 \mu \mathrm{Hz}$ up to $2000 \mu \mathrm{Hz}$ shows numerous low amplitude peaks which do not follow the spacing sequences of the low order modes. Although modes are not expected to follow the asymptotic spacing at low $n$, there seems to be too many of them for all to be of degree $l=1$ or 2 . In several other Kepler-observed sdBV stars Telting et al. (2014); Foster et al. (2015); Kern et al. (2017), multiplets in this frequency range indicate $3 \leq l \leq 9$ modes. A few frequencies are of instrumental nature ( $566.43 \mu \mathrm{Hz}$ and its multiples). In total, we found 24 frequencies in this region. But since we cannot see any signs of rotational splitting, there is no chance of identifying these modes.

Table 1: A list of frequencies found in the amplitude spectrum of KIC 10001893. Trapped modes are indicated with a "t" letter.

\begin{tabular}{cccccc}
\hline \hline ID & Freq[ $\mu \mathrm{Hz}]$ & Period[s] & Ampl[ppt] & $l$ & $n$ \\
\hline$f_{1}$ & 76.45610 & 13079.4 & 0.026 & & \\
$f_{2}$ & 77.52959 & 12898.3 & 0.098 & & \\
$f_{3}$ & 78.45598 & 12746.0 & 0.029 & & \\
$f_{4}$ & 79.71621 & 12544.5 & 0.023 & & \\
$f_{5}$ & 81.97998 & 12198.1 & 0.040 & & \\
$f_{6}$ & 83.93838 & 11913.5 & 0.021 & & \\
$f_{7}$ & 87.36142 & 11446.7 & 0.024 & & \\
$f_{8}$ & 89.08844 & 11224.8 & 0.048 & & \\
$f_{9}$ & 91.74985 & 10899.2 & 0.024 & & \\
$f_{10}$ & 96.93867 & 10315.8 & 0.056 & & \\
$f_{11}$ & 98.98050 & 10103.0 & 0.020 & & \\
$f_{12}$ & 102.17218 & 9787.4 & 0.027 & 1 & 36 \\
$f_{13}$ & 105.01995 & 9522.0 & 0.031 & 1 & 35 \\
$f_{14}$ & 107.83350 & 9269.3 & 0.031 & &
\end{tabular}

\begin{tabular}{|c|c|c|c|c|c|}
\hline$f_{15}$ & 108.18998 & 9243.0 & 0.036 & 1 & 34 \\
\hline$f_{16}$ & 111.64452 & 8957.0 & 0.023 & & \\
\hline$f_{17}$ & 114.41647 & 8740.0 & 0.062 & & \\
\hline$f_{18}$ & 114.71047 & 8717.6 & 0.038 & 1 & 32 \\
\hline$f_{19}$ & 116.91257 & 8553.4 & 0.060 & & \\
\hline$f_{20}$ & 118.33339 & 8450.7 & 0.032 & 1 & 31 \\
\hline$f_{21}$ & 120.49499 & 8299.1 & 0.022 & & \\
\hline$f_{22}$ & 122.07627 & 8191.6 & 0.019 & 1 & 30 \\
\hline$f_{23}$ & 123.88963 & 8071.7 & 0.023 & & \\
\hline$f_{24}$ & 125.85581 & 7945.6 & 0.048 & 1 & 29 \\
\hline$f_{25}$ & 126.89067 & 7880.8 & 0.034 & 1 & $\mathrm{t}$ \\
\hline$f_{26}$ & 130.55000 & 7659.9 & 0.041 & 1 & 28 \\
\hline$f_{27}$ & 132.47489 & 7548.6 & 0.033 & & \\
\hline$f_{28}$ & 135.32898 & 7389.4 & 0.098 & 1 & 27 \\
\hline$f_{29}$ & 140.52048 & 7116.4 & 0.223 & 1 & 26 \\
\hline$f_{30}$ & 141.47473 & 7068.4 & 0.029 & & \\
\hline$f_{31}$ & 145.73647 & 6861.7 & 0.036 & & \\
\hline$f_{32}$ & 146.10057 & 6844.6 & 0.088 & 1 & 25 \\
\hline$f_{33}$ & 152.06118 & 6576.3 & 0.098 & 1 & \\
\hline$f_{34}$ & 155.96730 & 6411.6 & 0.028 & & \\
\hline$f_{35}$ & 158.08277 & 6325.8 & 0.204 & 1 & 23 \\
\hline$f_{36}$ & 160.05890 & 6247.7 & 0.048 & 1 & 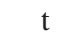 \\
\hline$f_{37}$ & 163.05499 & 6132.9 & 0.033 & & \\
\hline$f_{38}$ & 164.99199 & 6060.9 & 0.069 & & \\
\hline$f_{39}$ & 165.39587 & 6046.1 & 0.123 & 1 & 22 \\
\hline$f_{40}$ & 166.21235 & 6016.4 & 0.045 & & \\
\hline$f_{41}$ & 172.45839 & 5798.5 & 0.063 & & \\
\hline$f_{42}$ & 172.72052 & 5789.7 & 0.413 & 1 & 1 \\
\hline$f_{43}$ & 173.19016 & 5774.0 & 0.057 & & \\
\hline$f_{44}$ & 176.67844 & 5660.0 & 0.034 & & \\
\hline$f_{45}$ & 178.79492 & 5593.0 & 0.021 & & \\
\hline$f_{46}$ & 180.55430 & 5538.5 & 0.065 & 1 & 20 \\
\hline$f_{47}$ & 181.94388 & 5496.2 & 0.039 & 2 & 35 \\
\hline$f_{48}$ & 187.26591 & 5340.0 & 0.019 & 2 & 34 \\
\hline$f_{49}$ & 189.09310 & 5288.4 & 0.076 & 1 & 19 \\
\hline$f_{50}$ & 192.71535 & 5189.0 & 0.033 & 2 & 33 \\
\hline$f_{51}$ & 196.41734 & 5091.2 & 0.036 & & \\
\hline$f_{52}$ & 199.11196 & 5022.3 & 0.770 & 1 & 18 \\
\hline$f_{53}$ & 201.17486 & 4970.8 & 0.034 & 1 & $\mathrm{t}$ \\
\hline$f_{54}$ & 204.69571 & 4885.3 & 0.097 & 2 & 31 \\
\hline$f_{55}$ & 210.68155 & 4746.5 & 0.793 & 1 & 17 \\
\hline$f_{56}$ & 211.18878 & 4735.1 & 0.040 & 2 & 30 \\
\hline$f_{57}$ & 217.75580 & 4592.3 & 0.025 & 2 & 29 \\
\hline$f_{58}$ & 219.40891 & 4557.7 & 0.046 & 2 & $\mathrm{t}$ \\
\hline$f_{59}$ & 224.71910 & 4450.0 & 0.054 & 1 & 16 \\
\hline$f_{60}$ & 225.89681 & 4426.8 & 0.027 & 2 & 28 \\
\hline$f_{61}$ & 234.17558 & 4270.3 & 0.066 & 2 & 27 \\
\hline$f_{62}$ & 238.98288 & 4184.4 & 0.091 & 1 & 15 \\
\hline$f_{6}$ & 243.14335 & 4112.8 & 0.052 & 2 & 26 \\
\hline & 252.79336 & 3955.8 & 0.129 & 2 & 25 \\
\hline
\end{tabular}




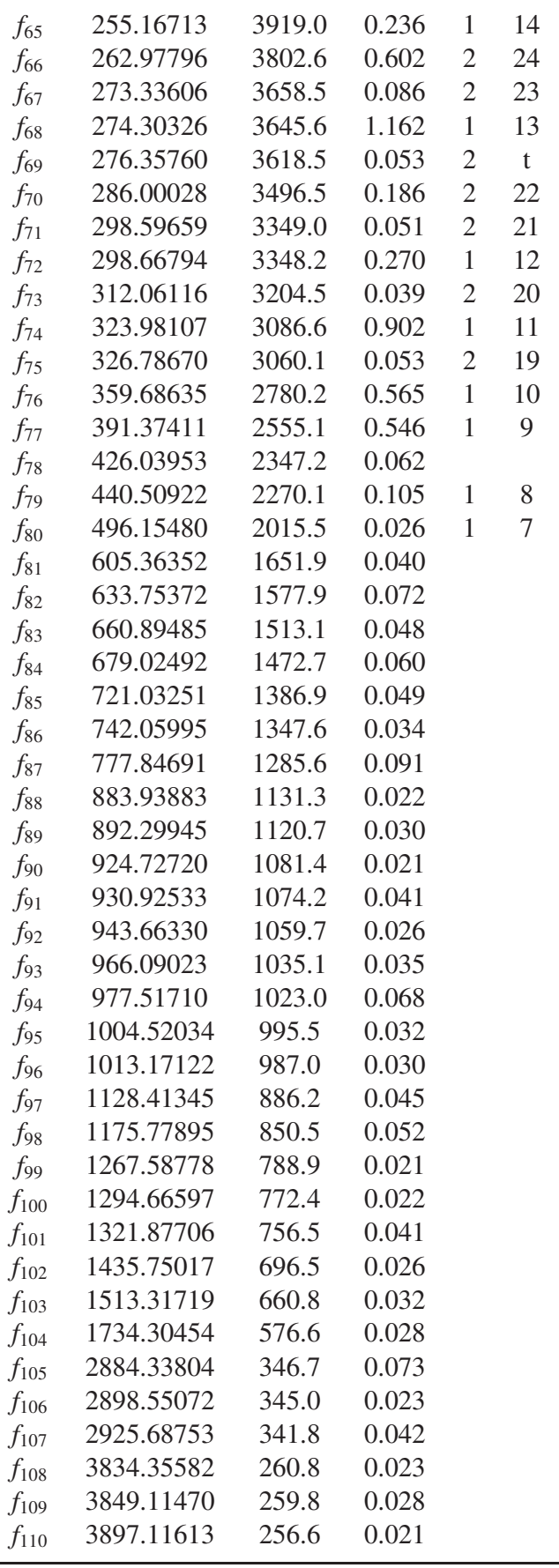

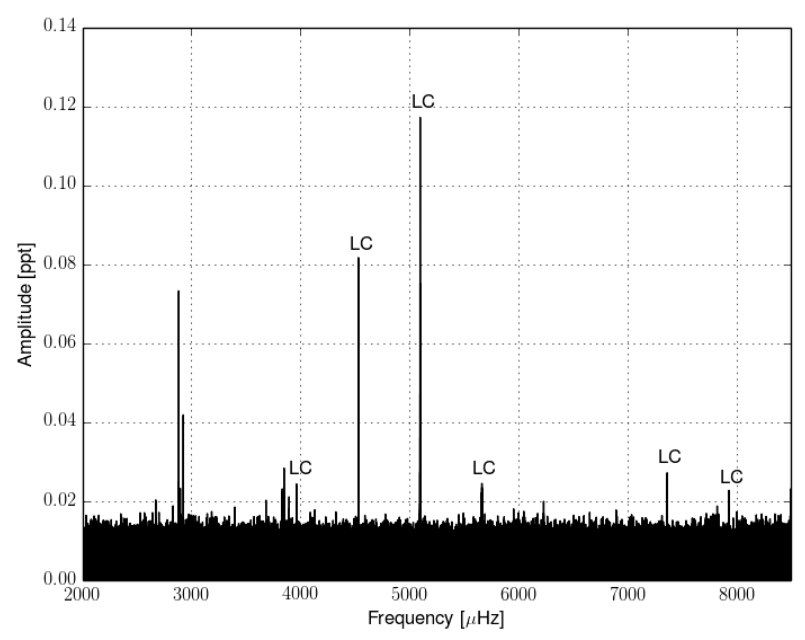

Figure 2. A close-up of the amplitude spectrum showing the high-frequency region.

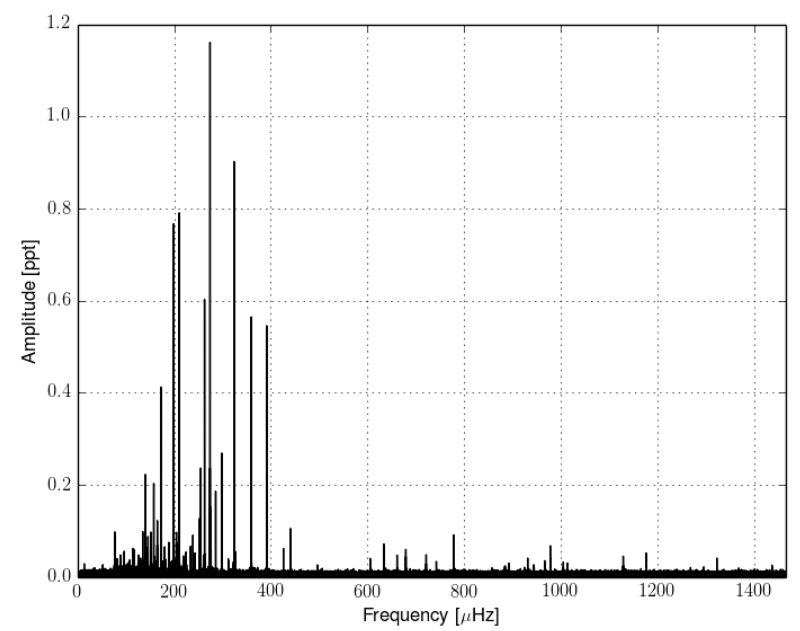

Figure 3. A close-up of the amplitude spectrum showing the low-frequency region

\section{MULTIPLETS}

The ultimate goal of our analysis was to identify modes to constrain theoretical models of this star. Mode identifications describe a mode's geometry, i.e. a radial order $n$, a modal degree $l$ and an azimuthal order $m$ of the mode. Information on these parameters is very crucial for calculation of stellar interior. Each mode is described by three numbers and therefore each mode adds three unknown parameters to the model. Since the more free parameters there are, the less reliable the model is, it is important to identify as many modes as possible.

In the presence of stellar rotation, non-radial modes of degree $l$ split into $2 l+1$ components differing in $m$ number. The azimuthal frequencies can be derived from the following equation 


$$
v_{n, l, m}=v_{n, l, 0}+\Delta v_{n, l, m}=v_{n, l, 0}+m \frac{1-C_{n, l}}{P_{\mathrm{rot}}}
$$

where $\Delta v_{n, l, m}$ is a rotational splitting. $\mathrm{P}_{\text {rot }}$ is a star's rotation period and $C_{n, l}$ is the Ledoux constant. We searched for multiplets among g-modes with a null result. The most likely explanation for this is that the inclination is too low, as was inferred by Silvotti et al. (2014). The authors concluded that the inclination may be as low as just a few degrees. If so, KIC 10001893 is another example of a pole-on oriented sdB star (such as KIC 8302197 Baran et al. $2015 b$ ). Other possibilities, like extremely slow rotation or selective driving of $m=0$ modes cannot be completely ruled out, though we consider this to be unlikely. The lack of multiplets prevents us from identifying azimuthal orders and determining a rotation rate for KIC 10001893.

If the absence of rotational splitting is due to extremely slow rotation, the time resolution of the Kepler data set provides a limit on the rotation period of Prot $\geq 715$ days. Here we used the FWHM of a few amplitude-stable modes $(0.00116 \mathrm{c} / \mathrm{d})$ to approximate the resolution needed to tell apart an $l>1$ multiplet, and we assumed the $\delta \mathrm{m}$ splitting of $\left(1-\mathrm{C}_{\mathrm{nl}}\right) / \mathrm{P}_{\text {rot }} \geq 0.83 / \mathrm{P}_{\text {rot }}$, expected for high-order $l>1$ g-modes. We examined the amplitudes of many of our modes and do not see a single characteristic time scale of amplitude variability longer than 715 days, which one may expect for unresolved beating between multiplet components. Therefore, if the lack of multiplets is due to very slow rotation, the rotation period must be well longer than the length of the dataset. This is in contrast with typical rotational rates of tens of days detected thus far (e.g. Baran 2012; Baran \& Winans 2012; Telting et al. 2012; Østensen et al. 2014; Reed et al. 2014; Foster et al. 2015).

For the other known case of an sdB pulsator which does not show any multiplets in a 3-year dataset, KIC 8302197, Baran et al. (2015b) argued that the lack of multiplets can be due to geometrical cancellation if the inclination is lower than a few degrees, but that the probability of having such low inclination angles is such that one would expect only a few such cases in every 1000 sdB stars. Finding two such cases in a sample of only $\sim 16$ well-studied sdB pulsators, is therefore somewhat problematic.

\section{PERIOD SPACING}

In the asymptotic limit $n>>l$, consecutive radial overtones are evenly spaced in period (e.g. Reed et al. 2011). For given $n$ and $l$ values, periods of consecutive overtones can be calculated from

$$
P_{l, n}=\frac{\Delta \Pi}{\sqrt{l(l+1)}} n+\epsilon_{l}
$$

where $\Delta \Pi$ is the reduced period spacing and $\epsilon_{l}$ is a constant offset smaller than $\Delta \Pi$. With this equation we define the asymptotic radial order of a mode, $n$, but note that this does not allow us to derive the true radial order of a mode, which is usually denoted $k$, since any trapped modes will be inserted into the the mode sequence. Thus, each trapped mode will bump the mode order $k$ by one relative to the asymptotic order $n$, at the point in the sequence where it occurs. The reduced period spacing $\Delta \Pi$ can be inferred from the échelle diagrams by converting the observed period spacings for each $l, \Delta P_{l}$, to reduced period, $\Delta \Pi=\sqrt{l(l+1)} \Delta P_{l}$. The offset $\epsilon_{l}$ can also be derived from the échelle diagrams as the mean value of the observed periods associated with a given $l$ modulo the period spacing. It was found from observations that the period spacing of $l=1$ modes is around $250 \mathrm{~s}$ (Reed et al. 2011), matching predictions from theoretical models (Charpinet et al. 2000). The spacings of $l=2$ modes are found to be around $145 \mathrm{~s}$, so that both produces reduced spacings of $\sim 350 \mathrm{~s}$. Period spacings for $l=3$ would translate to around $102 \mathrm{~s}$, but has never been detected.

Since pulsation periods depend on the local sound speed, chemical stratification in the resonant cavity will cause fluctuations in the observed period spacings at low $n$, but these should disappear at higher $n$ (Charpinet et al. 2014). For KIC 10001893 we derived the average of the spacing value for $l=1$ to be $268.0 \pm 0.5 \mathrm{~s}$, and $153 \pm 0.4 \mathrm{~s}$, respectively for $l=1$ and 2 . Our $l=1$ and 2 values match those of Reed et al. (2011). The sequence of $l=1$ modes is fairly complete, with 32 overtones identified. While $18 l=2$ overtones were identified, the sequence is nearly complete, only missing $n=32$. The identification of modal degrees is marked in Fig. 4 and provided in Table 1. KIC 10001893 is therefore another very successful case for mode identification in sdB stars, providing significant constraints for stellar modeling. Although the multiplets are not present, their absence actually makes it simpler since we can associate all modes with $m=0$ and avoid the ambiguity which exists when having incomplete multiplets, as was seen in KIC 10553698A (Østensen et al. 2014). A pole on orientation means that only the central components are visible, while an extremely slow rotation would imply that modes of different $m$ have the same frequency, effectively producing the same result. In either case, this does not reduce the value of the identification that we are able to make from asymptotic sequences alone. Although, we would have liked to use the multiplets for confirmation of our mode designations, having obtained those verifications from a large number of cases already, we are confident that the asymptotic sequences alone are sufficient to derive reliable mode identifications.

Reed et al. (2011) found period spacings between 230 and $270 \mathrm{~s}$ for the g-mode pulsators in the Kepler field. The spacing is determined by the size of the pulsation resonant cavity and is the first asteroseismic diagnostic that can be used to constrain pulsation models. For instance Schindler et al. (2015) found that models computed with MESA produced much larger cores when type II opacities were included and either overshoot or diffusion were turned on. Still, such large cores would reduce the size of the propagation cavity for g-modes and these are incompatible with all but the shortest period spacings found by Reed et al. (2011). Constantino et al. (2015) also concluded that their models tended to produce period spacings significantly below the average inferred from observations. The period spacing is therefore an important constraint for theoretical models, and since KIC 10001893 has one of the largest period spacings for known g-mode pulsators, it is an important case for modeling the extent of resonant cavities. 

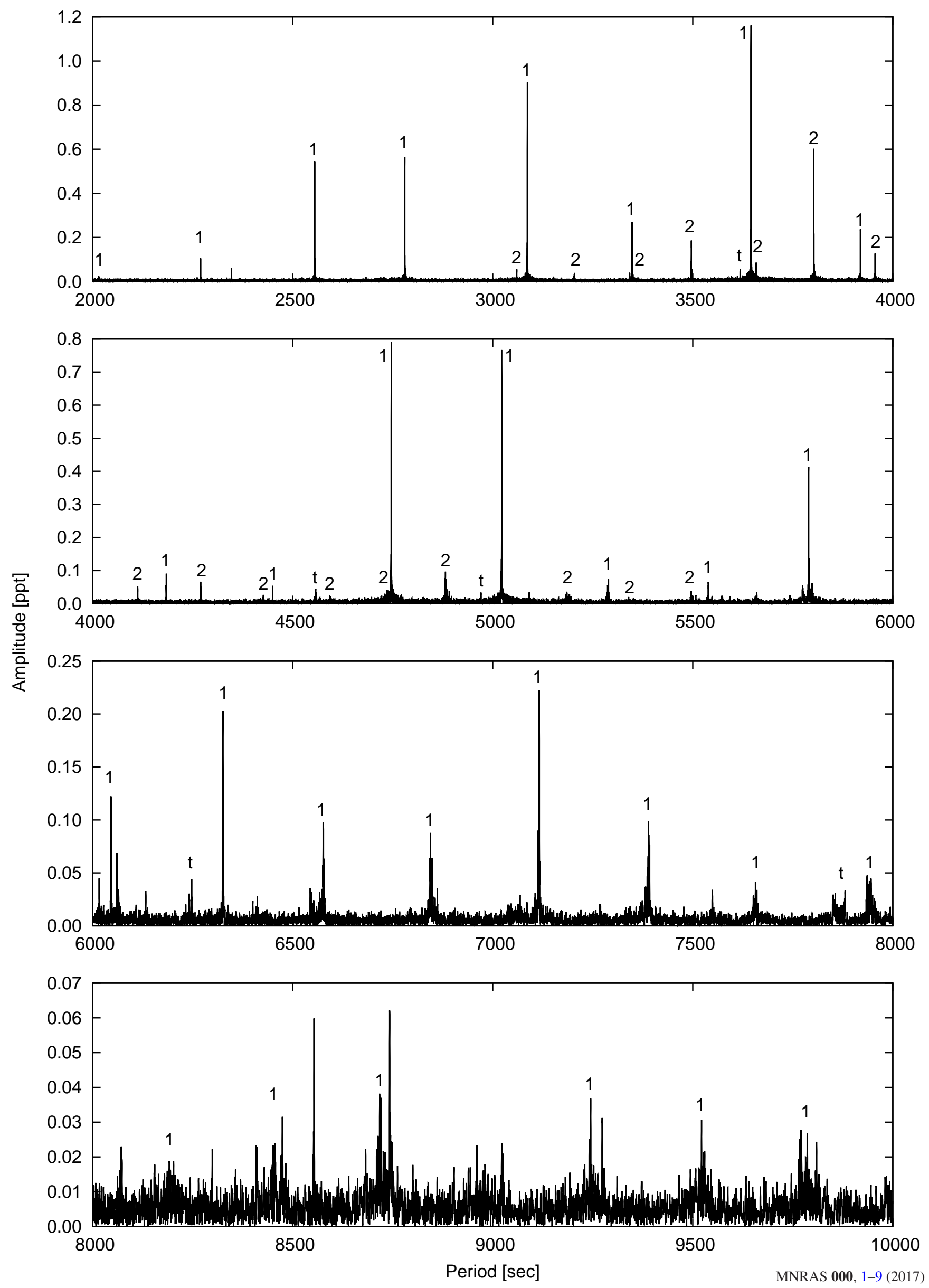


\section{MODE TRAPPING}

The asymptotic relation for g-modes, Eq. 2, holds for idealised stellar models with homogeneous composition. In that case the boundaries for a g-mode being a standing wave is a surface and, usually, a convective core. The consecutive overtones are perfectly spaced making an even sequence of modes of a given modal degree $l$, and the échelle diagrams would show a vertical ridge. However, real sdB stars are compositionally stratified. Composition discontinuities will produce steep gradients in density, which may act as a boundary for stellar pulsations. In the sdB stars, the two important composition transition regions are the $\mathrm{H} / \mathrm{He}$ boundary and the deep transition region between the helium mantle and the central convective core. In the models of Charpinet et al. (2014) it is the $\mathrm{H} / \mathrm{He}$ transition that produces the strongest trapping effect, while in the work of Ghasemi et al. (2017) strong trapping effects are produced by convective shells forming in the transition zone between the $\mathrm{C} / \mathrm{O}$-enhanced convective core and the radiative $\mathrm{He}$ mantle. These boundaries can cause partial reflection of the waves, or even act as an extra reflection surface which cause extra modes to be inserted into the otherwise roughly evenly spaced sequence of frequencies.

While the detection of trapped modes is in itself interesting, finding several of them and thereby being able to estimate the spacing between consecutive trapped modes, is even more useful since the spacing between consecutive trapped modes can be a particularly important diagnostic when comparing observations with theoretical models. When searching for trapped modes it is convenient to start with the identifications made in the échelle diagram for the $l=1$ and 2 sequences and plot them in a reduced-period diagram (where period, $P$, is converted to reduced period $\Pi=P \sqrt{\ell(\ell+1)}$ ). After conversion to reduced period, modes of the same order should fall roughly on top of each other. In the échelle diagram, trapped modes are shifted off the ridge and cannot therefore be assigned to a particular $l$. But with some trial and error it is possible to use the fact that the reduced-period differences should be the same for different $l$ to find solutions where two observed modes correspond to the same order $n$ for different $l$ and therefore line up in the reduced period diagram.

We calculated échelle diagrams for $l=1$ up to 5, though only $l=1$ and 2 overtones show evidence of systematic ridges (shown in Figs. 5 and 6). The diagram of $l=1$ modes shows a "hook" feature observed in some other sdBV stars (e.g. Baran \& Winans 2012). Such a feature appears in some models (e.g. Fig. 1 of Charpinet et al. 2014), and is related to the chemical stratification, but a quantitative explanation of this feature has not been presented, therefore we will postpone a discussion of this feature until models for this star are available. We used the asymptotic relation to define $n$ values for our modes, and these values are listed in Table 1 . We note that there may be a zero-point offset $n_{l}$ between the listed $n$ values and the actual mode order $k$, an offset that we cannot determine without the appropriate modeling. The trapped modes are effectively inserted between consecutive asymptotic radial orders, implying smaller period spacings between adjacent radial orders around the trapped mode.

The identification of two trapped modes between radial orders $n=22$ and 23 , and 28 and 29 , is very convincing as they are well matched in both the $l=1$ and 2 sequences (Fig. 7). The radial orders around the trapped modes agree between both modal degrees, as predicted by Charpinet et al. (2000) (their Eq. 30). A possible third trapped mode around $n=18$ is only present in the $l=1$ sequence, and as we could not recover the period-spacing relation for $l=2$ in that region, the significance of that trapped mode is much

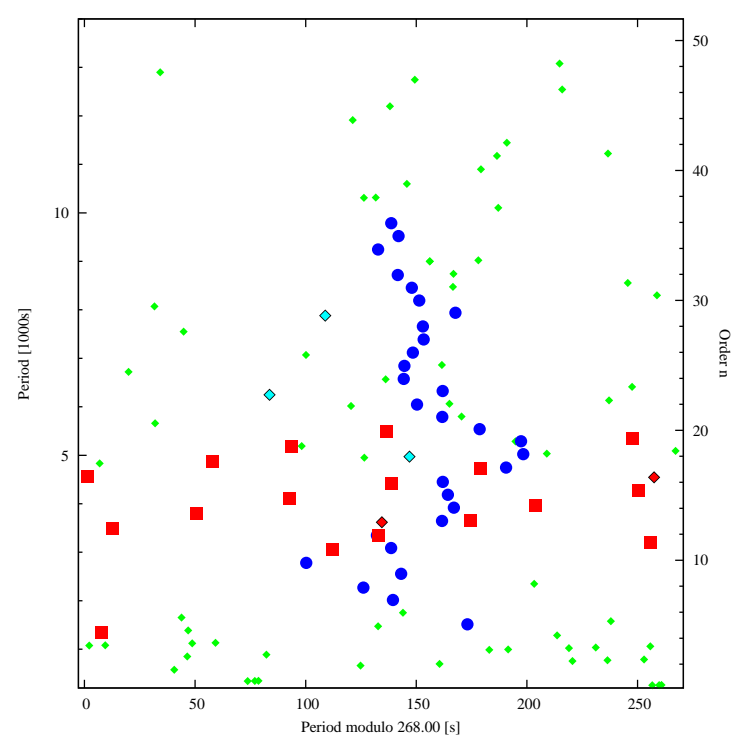

Figure 5. Échelle diagram folded over the average $l=1$ period spacing. Blue dots represent dipole modes, red squares quadrupole modes, while diamonds with black outlines represent trapped modes. Green diamonds show unidentified modes.

less convincing. Indeed, there are other unidentified modes which could be associated with trapped modes, but without multiplets, we have no way of preferring one over the other. For the overlap region, however, the match is exceptionally good, which gives us high confidence that those modes have been correctly identified.

The reduced-period spacing between the trapped modes is roughly $2000 \mathrm{~s}$ and this observation agrees with the third trapped mode. In the models of Charpinet et al. (2000), this spacing can be taken as a direct measure of the mass of the $\mathrm{H}$ envelope, and it was estimated by Charpinet et al. (2000) that it should be of the order of $2000 \mathrm{~s}$, in excellent agreement with our observation. Charpinet et al. (2000) found that for some trapped modes the minimum in period spacing does not correspond to the minimum of the kinetic energy, as expected, but to the maximum instead. Such trapped modes will not be spaced by around $2000 \mathrm{~s}$, but somewhat closer, and will not overlap between modal degrees. We found two spacings, both close to $2000 \mathrm{~s}$. According to Charpinet et al. (2000), our observations suggest that only the $\mathrm{He} / \mathrm{H}$ transition zone contributes to the trapping, while the impact from the other, C$\mathrm{O} / \mathrm{He}$, zone is negligible. Having the spacing measured precisely, we could compare it with the models to find the location of the base of the $\mathrm{H}$ envelope and its mass. This task will be done as soon as the specific model of this star is available. 


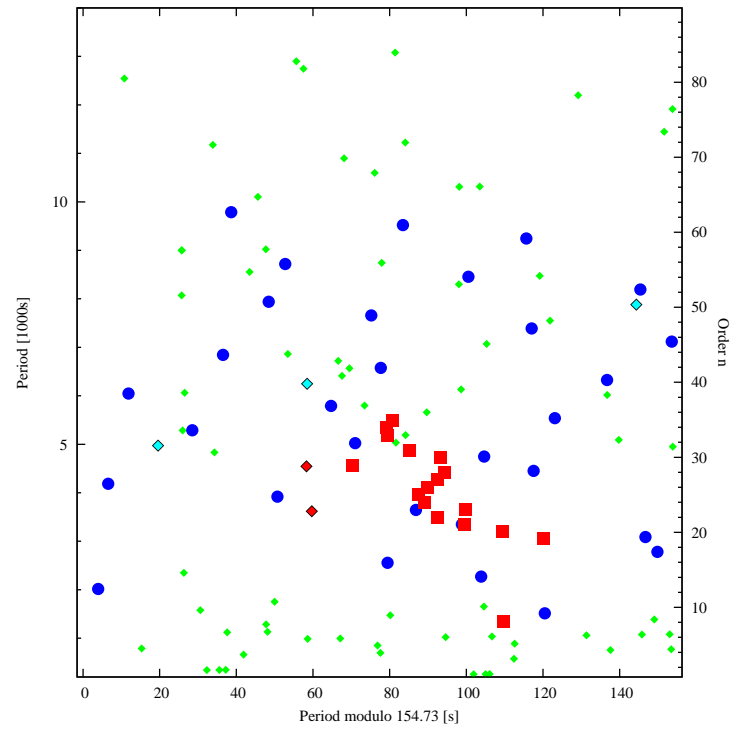

Figure 6. Same as in Fig. 5 but for quadrupole modes.

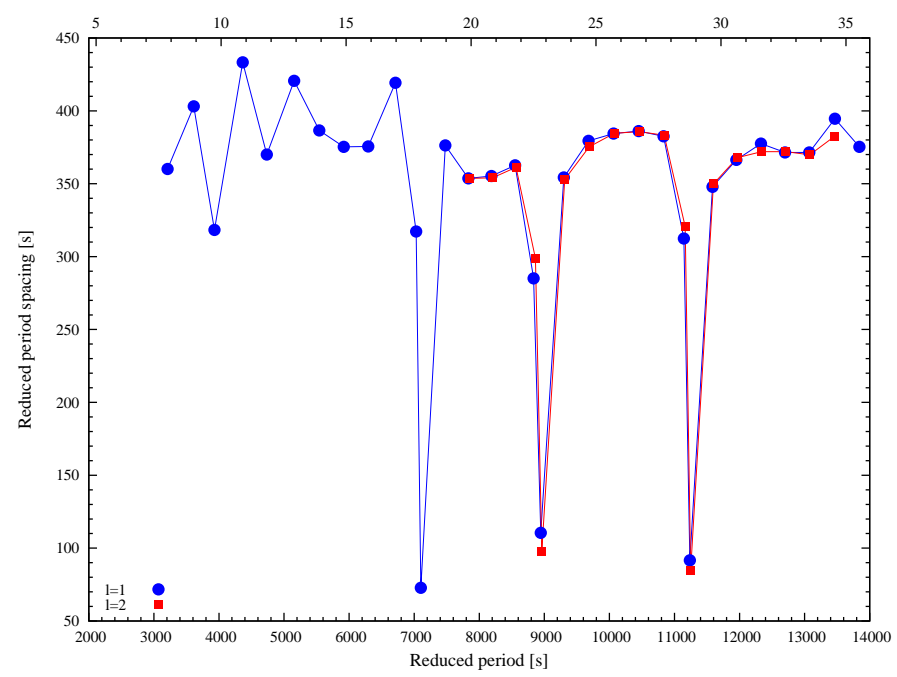

Figure 7. Reduced Period Diagram. Reduced period is defined as $P \sqrt{\ell(\ell+1)}$

\section{SUMMARY AND CONCLUSIONS}

We have analyzed KIC 10001893, a V1093 Her type sdBV star observed with the Kepler spacecraft. The data consists of a nearly uninterrupted $1051 \mathrm{~d}$ sequence with 1-minute cadence. An amplitude spectrum uncovered a rich content of 110 pulsation modes. We recovered all 27 frequencies of (Baran et al. 2011), and while amplitudes vary somewhat, those frequencies are consistent between Q3.3 and this much longer data set. The amplitude spectrum shows both g- and p-modes, but g-modes are strongly favored, with only 6 p-modes all below 0.1 ppt in amplitude. So while KIC 10001893 is clearly a hybrid pulsator, it is quite different from the DW Lyn-type hybrid pulsators which show p- and g-modes in similar amounts. Hybridity can be an important feature which provides simultaneous constraints both on core and envelope parameters.

We find that KIC 10001893 is quite an enigmatic object with respect to rotationally split multiplets, a feature normally easily seen in similar stars. We found no multiplets among either $\mathrm{p}$ - or g-modes, with the most likely explanation being a very low inclination of the star. This would then be the second sdBV star oriented pole on (Baran et al. 2015b). We identified the modal degree for 50 of the detected g-modes, which is about half of the modes, and includes all of the modes with amplitudes higher than $0.1 \mathrm{ppt}$. Out of those 50 modes, 32 are dipole modes and 18 are quadrupole modes. We calculated échelle diagrams for $l=1$ and 2 (Fig. 5 and 6) and they show wavy excursions from a straight ridge, which are indicative of non-uniform density profiles in the stellar interior. This feature is similar to ones detected in other Kepler-observed sdBV stars (see e.g. Baran \& Winans 2012; Østensen et al. 2014; Foster et al. 2015; Baran et al. 2017).

After identifying almost complete sequences of consecutive radial orders for both $l=1$ and 2, including several trapped modes, we were able to construct a reduced-period diagram showing almost perfect alignment of the two sequences. The location of the trapped modes, and particularly the spacing between them, provides a useful tool to examine the stellar interior and test various modeling prescriptions against each other. The trapping can be caused by either the transition region of $\mathrm{He} / \mathrm{H}$ at the base of the H-rich envelope, or deeper trapping regions associated with the transition region between the convective and radiative part of the core. Fig. 7 shows three trapped modes, with two of them overlapping between $l=1$ and 2 sequences. This pattern is almost identical to the one seen in KIC 10553698A, but with all three trapped modes shifted to slightly higher periods. The overlap between the two sequences is so close that in spite of the lack of multiplets as a crosscheck for mode identifications, we are confident of our conclusions, at least for the region of overlap. We consider KIC 10001893 to be one of the most prominent sdB stars for applying asteroseismology to infer the physics of the deep interiors of core-helium burning stars.

\section{ACKNOWLEDGEMENTS}

ASB gratefully acknowledges financial support from the Polish National Science Center under project No. UMO2011/03/D/ST9/01914.

\section{REFERENCES}

Baran A. S., 2012, Acta Astron., 62, 179

Baran A. S., 2013, Acta Astron., 63, 203

Baran A. S., Winans A., 2012, Acta Astron., 62, 343

Baran A., Pigulski A., Kozieł D., Ogłoza W., Silvotti R., Zoła S., 2005, MNRAS, 360, 737

Baran A. S., et al., 2011, MNRAS, 414, 2871

Baran A. S., Koen C., Pokrzywka B., 2015a, MNRAS, 448, L16

Baran A. S., Telting J. H., Németh P., Bachulski S., Krzesiński J., 2015b, A\&A, 573, A52

Baran A. S., Reed M. D., Østensen R. H., Telting J. H., Jeffery C. S., 2017, A\&A, 597, A95

Charpinet S., Fontaine G., Brassard P., Dorman B., 1996, ApJ, 471, L103

Charpinet S., Fontaine G., Brassard P., Chayer P., Rogers F. J., Iglesias C. A., Dorman B., 1997, ApJ, 483, L123

Charpinet S., Fontaine G., Brassard P., Dorman B., 2000, ApJS, 131, 223

Charpinet S., et al., 2011, A\&A, 530, A3

Charpinet S., Brassard P., Van Grootel V., Fontaine G., 2014, in van Grootel V., Green E., Fontaine G., Charpinet S., eds, Astronomical Society of the Pacific Conference Series Vol. 481, 6th Meeting on Hot Subdwarf Stars and Related Objects. p. 179 
Constantino T., Campbell S. W., Christensen-Dalsgaard J., Lattanzio J. C., Stello D., 2015, MNRAS, 452, 123

Fontaine G., Brassard P., Charpinet S., Green E. M., Chayer P., Billères M., Randall S. K., 2003, ApJ, 597, 518

Foster H. M., Reed M. D., Telting J. H., Østensen R. H., Baran A. S., 2015, ApJ, 805, 94

Ghasemi H., Moravveji E., Aerts C., Safari H., Vučković M., 2017, MNRAS, 465, 1518

Han Z., Podsiadlowski P., Maxted P. F. L., Marsh T. R., Ivanova N., 2002, MNRAS, 336, 449

Heber U., 2016, PASP, 128, 082001

Kern J. W., Reed M. D., Baran A. S., Østensen R. H., Telting J. H., 2017, MNRAS, 465, 1057

Kilkenny D., Koen C., O'Donoghue D., Stobie R. S., 1997, MNRAS, 285,640

Koen C., O'Donoghue D., Pollacco D. L., Charpinet S., 1999, MNRAS, 305,28

Østensen R. H., et al., 2010, MNRAS, 409, 1470

Østensen R. H., et al., 2011, MNRAS, 414, 2860

Østensen R. H., Telting J. H., Reed M. D., Baran A. S., Nemeth P., Kiaeerad F., 2014, A\&A, 569, A15

Reed M. D., et al., 2011, MNRAS, 414, 2885

Reed M. D., Foster H., Telting J. H., Østensen R. H., Farris L. H., Oreiro R., Baran A. S., 2014, MNRAS, 440, 3809

Schindler J.-T., Green E. M., Arnett W. D., 2015, ApJ, 806, 178

Schuh S., Huber J., Dreizler S., Heber U., O’Toole S. J., Green E. M., Fontaine G., 2006, A\&A, 445, L31

Silvotti R., et al., 2014, A\&A, 570, A130

Telting J. H., et al., 2012, A\&A, 544, A1

Telting J. H., et al., 2014, A\&A, 570, A129

Van Grootel V., Charpinet S., Brassard P., Fontaine G., Green E. M., 2013, A\&A, 553, A97

Yong H., Demarque P., Yi S., 2000, ApJ, 539, 928

This paper has been typeset from a $\mathrm{T}_{\mathrm{E}} \mathrm{X} / \mathrm{LT}_{\mathrm{E}} \mathrm{X}$ file prepared by the author. 